descriptive comparisons, differences in proportions were determined by Fisher's exact test, and ANOVA determined differences in means for continuous variables. Multivariate linear models estimated the effect of RA on AMH. In women with RA, the effect of RA medication use on $\mathrm{AMH}$ and anovulation was explored.

Results: There were 75 RA patients (83\% Caucasian, 4\% Hispanic, $77 \%$ with at least a college education) and 75 controls (64\% Caucasian, 5\% Hispanic, $88 \%$ with at least a college education). The majority of RA patients were married $(60 \%)$, compared to $31 \%$ of controls. The mean age of both RA patients and controls was 32 years. Mean AMH in RA patients was 3.0 (SD: 2.6) compared to 3.9 (SD: 3.9 ) in controls (p-value: 0.1 ). In linear regression models adjusted for age, hormonal contraceptives and race (nonwhite vs. white), RA patients had a lower AMH than healthy controls $(\beta:-1.05 ; 95 \% \mathrm{Cl}:-2.09,-0.005 ; \mathrm{p}=0.05)$. There was no observed difference in the proportion of RA patients and controls with anovulation (19\% in RA and $21 \%$ in controls). Infertility was reported by $12 \%$ of RA patients and $7 \%$ of controls $(\mathrm{p}=0.4)$.

Among RA patients, $81 \%$ reported having ever used methotrexate (MTX). The mean AMH for MTX users was 2.8 (SD: 2.4) compared to 4.0 (SD: 3.1) in never users $(\mathrm{p}=0.1)$. In linear regression models adjusted for age, hormonal contraceptives and race, RA patients who had ever taken MTX had a lower AMH than those who had never taken MTX $(\beta:-1.49 ; 95 \% \mathrm{Cl}:-2.83,-0.15 ; \mathrm{p}=0.03)$. However, when the cumulative dose of MTX was analyzed, there was no effect of cumulative MTX and AMH. Ever use of prednisone or NSAIDs did not appear to affect AMH levels in RA patients. Methotrexate, prednisone, and NSAIDs use did not have an observed effect on anovulation.

Conclusions: In this cross-sectional study, women with RA appeared to have a lower $\mathrm{AMH}$ level than healthy controls, suggesting ovarian reserve may be lower in these patients. In RA patients, previous use of methotrexate was associated with lower $\mathrm{AMH}$, although no dose response of cumulative methotrexate exposure was observed. We did not observe a difference in anovulation between RA patients and controls. This suggests women with RA may have reduced fertility for reasons other than anovulation.

Acknowledgements: This study was funded by Pfizer.

Disclosure of Interest: M. Clowse Grant/research support from: Pfizer, Janssen, Consultant for: UCB, G. McDaniel: None declared, A. Eudy: None declared DOI: 10.1136/annrheumdis-2017-eular.6376

\section{FRI0158 CAROTID ENLARGEMENT, BRACHIAL ARTERY FLOW-MEDIATED VASODILATATION AND SERUM LEVELS OF VON WILLEBRAND FACTOR IN RHEUMATOID ARTHRITIS}

M. Veselinovic, A. Tomic-Lucic, V. Grbovic, V. Jakovljevic. KC Kragujevac, Kragujevac, Serbia

\section{Background:}

In rheumatoid arthritis (RA), higher mortality is mainly due to cardiovascular disease, as a consequence of accelerated atherosclerosis found in this diseases (1). Finding methods for assessing vascular dysfunction during the early stages of the disease is important, particularly in patient groups at high CV risk (2).

The use of noninvasive imaging techniques may help identify high-risk individuals who may benefit from active therapy to prevent clinical disease. Two of them, brachial ultrasonography to determine the presence of endothelial dysfunction and carotid ultrasonography to assess carotid intima-media wall thickness (IMT) have been found to be useful in the assessment of the cardiovascular risk of patients with RA (3). Prothrombotic markers have been shown to be able to predict cardiovascular risk in patients with RA (4).

Objectives: The first aim of the study was to investigate whether early signs of atherosclerosis and endothelial dysfunction, as measured by IMT and brachial artery flow-mediated (FMD) vasodilatation, were present in patients with RA compared with controls. The second aim was to analyze correlation between serum levels of vWf and IMT and FMD in patients with RA.

Methods: Fifty-two patients with RA and 30 matched healthy controls without clinically evident CV disease were studied. Brachial and carotid ultrasonography was performed to determine FMD and IMT, respectively. We also assayed immunological, inflammatory and metabolic laboratory markers.

Results: IMT was significantly higher in RA patients $(1.00 \pm 0.16 \mathrm{~mm})$ patients than in controls $(0.89 \pm 0.13 \mathrm{~mm})(P=0.001)$. FMD\% was significantly lower in RA $(9.16 \pm 7.03)$ as compared to controls $(12.60 \pm 5.49)(p=0.005)$. It showed statistically higher values of $\mathrm{VWF}$ in the RA patients compared to the control group $(\mathrm{p}=0.01)$.

Patients with RA is divided into two groups according to the value of the IMT, below $9 \mathrm{~mm}$ and $0.9 \mathrm{~mm}$ and above. vWFact is statistical significantly higher in the group with thicker IMT $(p=0.046)$, as well as the percentage of men $(p=0.030)$. Other parameters did not show statistical significance in relation to the value of the IMT.

Conclusions: In patients with RA, increased serum levels of VWF and impaired FMD and IMT, indicating endothelial dysfunction and accelerated atherosclerosis. IMT measurement is the first candidate to evaluate against conventional evaluation of cardiovascular risk in prospective studies.

References:

[1] Dessein PH, Joffe BI, Singh S. Biomarkers of endothelial dysfunction, cardiovascular risk factors and atherosclerosis in rheumatoid arthritis. Arthritis Res Ther 2005;7:634-643.

[2] Tousolis D, Antoniades C, Bosinakou E et a. Differences in inflammatory and thrombotic markers between unstable angina and acute myocardial infarction. Int J Cardiol 2007;115:203-207.

[3] Fuchs I, Frossard M, Spiel A, Riedmuller E, Laggner AN, Jilma B. Platelet function in patients with acute coronary syndrome (ACS) predicts recurrent ACS. J Thromb Haemost 2006;4:2547-2552.

[4] Jonsson SW, Backman C, Johnson $O$ et al. Increased prevalence of atherosclerosis in patients with medium term rheumatoid arthritis. J Rheumatol 2001;28:2597-2602.

Disclosure of Interest: None declared

DOI: 10.1136/annrheumdis-2017-eular.1216

\section{FRI0159 INFECTION AND MALIGNANCY ARE NOW THE MAJOR CAUSES OF DEATH IN AGGRESSIVELY TREATED RHEUMATOID ARTHRITIS PATIENTS}

N. Chaplin ${ }^{1}$, J. McNally ${ }^{2}$, J. Kitchen ${ }^{2} .{ }^{1}$ St George's University of London, London; ${ }^{2}$ Rheumatology, Royal Berkshire NHS Foundation Trust, Reading, UK

Background: Patients with rheumatoid arthritis (RA) have an increased mortality compared to the general population. Previous studies have shown that this is attributable to cardiovascular and respiratory disease. Over recent years earlier and more aggressive treatment with higher dosage of methotrexate and the earlier use of biologic drugs has improved disease outcomes. The effect on cause of death is unknown. In this retrospective cohort study we found the major cause of death was infection followed by malignancy and found no correlation with seropositivity or gender.

Objectives: To analyse the causes of death in RA patients treated with aggressive disease modifying anti rheumatic drugs (DMARD) and Biologic therapies in Berkshire, UK.

Methods: Patients with RA who died between 2010 and 2016 were identified using the DAWN software DMARD monitoring database. A cohort of 3106 patients with RA are monitored using DAWN software in Berkshire, UK. The causes of death were identified from medical records, general practice records or the local coroners office.

Results: 198 patients on DAWN monitoring died during the 6 year study period. Treatment details and cause of death was identified for 131 RA patients. $71 \%$ were seropositive for rheumatoid factor and $61 \%$ were female.

91 patients $(69 \%)$ were treated with methotrexate, 28 hydroxychloroquine $(21 \%)$, 14 sulphasalazine $(21 \%)$ and 7 with leflunomide $(5 \%)$. The majority of patients (81) were on monotherapy (61\%), 32 were on 2 DMARDS $(24 \%)$ and only one was on triple therapy.

4 patients with RA who died were on biologic monotherapy, 10 were treated with biologic and combination DMARD. The most commonly prescribed biologics were etanercept $(35 \%)$ and rituximab $(35 \%)$.

The leading causes of death in this cohort were pneumonia (39 patients $29 \%$ ), cerebrovascular disease (16 patients $12 \%$ ), septicaemia (11 patients $8 \%$ ) and lung cancer (6 patients $4 \%)$.

Infection accounted for 57 patients' deaths (43\%) followed by malignancy in 24 patients $(18 \%)$. Cerebrovascular disease (20 patients, $15 \%)$ and cardiovascular disease (13 patients, $9 \%$ ) were less frequent causes of death in our cohort.

Comorbidity data for the cohort was recorded premortem. 49 patients $(37 \%)$ had cardiovascular disease of any kind. $25 \%$ had respiratory disease and $23 \%$ had an endocrine comorbidity (predominantly diabetes).

Conclusions: In our large cohort of aggressively treated RA patients, infection followed by malignancy and not cardiovascular disease, was the leading cause of death. Larger prospective studies will be required to see if cumulative drug toxicity of more aggressive early treatment improves outcome from RA but changes mortality from comorbidities over time.

Disclosure of Interest: None declared

DOI: 10.1136/annrheumdis-2017-eular.5442

\section{FRI0160 EVALUATION OF INFLAMMATORY CARDIOVASCULAR (CV) RISK FACTORS IN PRE- AND POST-MENOPAUSAL FEMALE PATIENTS WITH RHEUMATOID ARTHRITIS (RA)}

O.O. Garmish, V. Levchenko, V. Kovalenko. NSC "Institute of Cardiology named by M.D.Strazhesko NAMS of Ukraine", Kiev, Ukraine

Objectives: To assess inflammatory factors and peripheral vessels involvement as markers of cardiovascular risk in female patients with RA

Methods: 105 female patients who fulfill ACR/EULAR 2010 criteria were examined. Laboratory assessments consisted of biochemistry and hematology analysis, measuring of CRP level, rheumatoid factor, anti-CCP level, total cholesterol, HDL, LDL, thyroglobulin, apoliprotein, A1, apoliprotein B, uric acid, $\mathrm{HbA1c}$, microalbuminuria. DAS28 was used in characterizing RA activity. CV risk was defined per mSCORE. Tibial artery and carotid artery ultrasonography examination included the measurement of cIMT in 3 points, detection of focal plaques in the extracranial carotid tree, blood flow velocity and morphology of the intima was performed

Results: $83.3 \%$ reproductive age patients were without CV risk, $11.1 \%$ experienced middle level and $5.6 \%$ low level of CV risk on mSCOR. In $96.1 \%$ postmenopausal patients moderate, high and very high CV risk was detected. According to multiple logistic regression analysis we identified CV risk factors: 
high CRP and DAS28, swollen joint count, LDL cholesterol level, menopause, thickness of CIMT and tibial artery. Were identify a significant correlation between tibial artery thickness and age, BMI, RA duration, systolic and diastolic blood pressure, cholesterol, menopause $(p<0.01, \times 2=26.18)$. Also, we identified a significant relationship between tibial artery thickness and changes of the intima morphology $(x 2=31,64 ; p<0,01)$, carotid plaques $(x 2=26,179 ; p<0,01)$, sclerosis of the heart valves $(x 2=25,78 ; p<0,01)$. A less significant relationship was between $\operatorname{cIMT}(x 2=8,507 ; p<0,01)$. The DAS28, age $(p<0.001)$, thickness of cIMT and tibial artery $(p<0.001)$ are predictive factors for the development of carotid plaques. The predictive factors for tibial artery thickness in female patients $<45$ age are: high CRP $(p<0.004)$ and DAS28 $(p<0.03)$, anti-CCP positivity $(p<0.04)$, Vps internal carotid artery $(p<0.04)$

Conclusions: The results of our study indicate that high CRP and DAS28 score, swollen joint count, LDL cholesterol level, menopause, thickness of cIMT and tibial artery associated with the increased cardiovascular risk. Tibial artery thickness and Vps internal carotid artery may be assessed like the new predictive factors of $\mathrm{CV}$ diseases in RA female patient

Acknowledgements: None

Disclosure of Interest: None declared

DOI: 10.1136/annrheumdis-2017-eular.6388

\section{FRI0161 NON-INVASIVE ASSESSMENT OF MYOCARDIAL PERFUSION IN PATIENTS WITH RHEUMATOID ARTHRITIS}

P. Anyfanti ${ }^{1}$, N. Koletsos ${ }^{1}$, A. Triantafyllou ${ }^{1}$, S. Chatzimichailidou ${ }^{2}$, G. Triantafyllou ${ }^{1}$, P. Panagopoulos ${ }^{1}$, E. Gkaliagkousi ${ }^{1}$, S. Aslanidis ${ }^{2}$, S. Douma ${ }^{1} .{ }^{1}$ rd Department of Internal Medicine, Papageorgiou Hospital; ${ }^{2} 2 n d$ Propedeutic Department of Internal Medicine, Hippokration Hospital, Aristotle University of Thessaloniki, Thessaloniki, Greece

Background: Cardiac involvement among patients with rheumatoid arthritis (RA) is common, potentially life-threatening, but often underdiagnosed at presymptomatic stages. Subendocardial viability ratio (SEVR) reflects microvascular coronary perfusion, as it correlates with the ratio of subepicardial to subendocardial blood flow, that can be non-invasively estimated by applanation tonometry. Although it has been studied as a surrogate measure of myocardial perfusion in high-cardiovascular risk populations, it remains unclear whether it is affected in RA patients.

Objectives: The purpose of the study was to compare SEVR between RA patients and healthy controls. We additionally sought predictors of SEVR in RA among a wide range of disease-related parameters, hemodynamic factors, and markers of atherosclerosis, arteriosclerosis, and endothelial dysfunction.

Methods: Consecutive patients with RA and healthy, nontreated volunteers were recruited. SEVR was estimated from applanation tonometry with the Sphygmocor device (AtCor Medical, Sydney, Australia), which was also used to evaluate arterial stiffness (aortic index, Alx; pulse wave velocity, PWV). In the RA group, carotid atherosclerosis was additionally evaluated by ultrasound (carotid intima-media thickness, cIMT); cardiac and hemodynamic parameters by impedance cardiography, and endothelial dysfunction by measurement of asymmetric dimethylarginine (ADMA) in serum samples.

Results: A total of 122 participants, 91 RA patients and 31 controls, were studied. SEVR was significantly lower among RA patients compared to controls (141.4 \pm 21.9 vs $153.1 \pm 18.7 \%, p=0.005)$, and the same was observed when the subgroup of RA patients without cardiovascular comorbidities $(n=29)$ was studied separately $(139.7 \pm 21.7$ vs $153.1 \pm 18.7 \%, p=0.013)$. In the univariate analysis, SEVR significantly correlated with cardiac and hemodynamic parameters, but not with PWV, Alx, CIMT, ADMA, or disease-related parameters. In the linear regression analysis accounting for sex, statin use, markers of atherosclerosis, cardiac, and hemodynamic parameters, female gender $(p=0.007)$, blood pressure $(p=0.028)$, heart rate $(p=0.025)$, cholesterol levels $(p=0.008)$, cardiac index $(p<0.001)$, and left ventricular ejection time $(p=0.004)$ were identified as independent predictors of SEVR among patients with RA.

Conclusions: Patients with RA exhibit lower values of SEVR compared to healthy individuals, suggesting a disturbed balance between oxygen supply and demand that might provide an additional pathophysiological link for the increased cardiovascular burden in RA. Cardiac and hemodynamic parameters, rather than markers of atherosclerosis, arteriosclerosis, and endothelial dysfunction, may be useful as predictors of impaired myocardial perfusion in RA.

References:

[1] Buckberg GD, et al. Experimental subendocardial ischemia in dogs with normal coronary arteries. Circ Res 1972;30:67-81.

[2] Sarnoff SJ, et al. Hemodynamic determinants of oxygen consumption of the heart with special reference to the tension-time index. Am J Physiol 1958;192:148-56.
[3] Tsiachris D, et al. Subendocardial viability ratio as an index of impaired coronary flow reserve in hypertensives without significant coronary artery stenoses. J Hum Hypertens 2012;26:64-70.

Disclosure of Interest: None declared

DOI: 10.1136/annrheumdis-2017-eular.4903

\section{FRI0162 ANTIBODY RESPONSE TO 13-VALENT PNEUMOCOCCAL CONJUGATE VACCINE IS NOT IMPAIRED IN PATIENTS WITH RHEUMATOID ARTHRITIS (RA) OR SJOGRENS SYNDROME WITHOUT DMARD TREATMENT COMPARED TO CONTROLS}

P. Nived $^{1,2}$, J. Nagel ${ }^{1}$, T. Saxne ${ }^{1}$, P. Geborek ${ }^{1}$, T. Mandl ${ }^{3}$, L. Skattum ${ }^{4,5}$, M.C. Kapetanovic ${ }^{1} .{ }^{1}$ Department of Clinical Sciences Lund, Section of Rheumatology, Skåne University Hospital, Lund University, Lund; ${ }^{2}$ Clinic for Infectious Diseases, KRYH, Kristianstad; ${ }^{3}$ Department of Clinical Sciences Malmö, Section of Rheumatology, Skåne University Hospital, Lund University, Malmö; ${ }^{4}$ Clinical Immunology and Transfusion Medicine, Region Skåne;

${ }^{5}$ Department of Laboratory Medicine, Section of Microbiology, Immunology and Glycobiology, Lund University, Lund, Sweden

Objectives: To investigate if antibody response and functionality of antibodies following immunization with 13-valent pneumococcal conjugate vaccine (PCV13) is impaired in patients with rheumatoid arthritis (RA) and primary Sjogrens syndrome (pSS) without treatment, with methotrexate (MTX) or hydroxychloroquine (HCQ), compared to healthy controls.

Methods: In total, 61 patients with RA (51 without DMARD and 10 on MTX), 23 patients with pSS (18 without DMARD and 5 on $\mathrm{HCQ}$ ) and 49 controls were vaccinated with a single dose $(0.5 \mathrm{ml})$ PCV13 intramuscularly. Pre- and postvaccination serotype-specific antibody concentrations for pneumococcal serotypes $6 \mathrm{~B}$ and $23 \mathrm{~F}$ and functionality of antibodies $(23 \mathrm{~F}$ ) were determined in serum samples taken immediately before and 4-6 weeks after vaccination using ELISA and opsonophagocytic activity (OPA) assay, respectively. Proportions of individuals with positive antibody response (i.e. $\geq 2$-fold increase from prevaccination concentrations) for both serotypes were calculated and groups were compared using Chi2 test. Percentage change (pre- to postvaccination) in OPA was calculated and groups were compared using Mann-Whitney $\mathrm{U}$ test.

Results: Pre- to postvaccination antibody concentrations (Table) increased significantly for both serotypes and in both patient and control groups $(p<0.001)$. Antibody response to serotypes $6 \mathrm{~B}$ and $23 \mathrm{~F}$ was decreased in patients with $\mathrm{RA}$ on MTX treatment (both $p<0.01$ ), but not in RA without DMARD or pSS with or without $\mathrm{HCQ}$, compared to controls (Figure). Proportions of antibody responders to both serotypes did not differ significantly between groups RA without DMARD $(51 \%)$, pSS $(48 \%)$ and controls (55\%). After vaccination, OPA increased in groups RA without DMARD $(p<0.001)$, pSS without DMARD $(p=0.01)$ and controls $(p<0.001)$, but did not change significantly in patients with RA on MTX and pSS on HCQ. The mean percentage change in OPA was lower in RA on MTX $(1.8 \%$, $p=0.01)$, RA without DMARD $(8.9 \%, p<0.01)$ and $\mathrm{pSS}$ on $\mathrm{HCQ}(-1.5 \%, p=0.01)$, but not in pSS without DMARD (11.0\%), compared to controls (17.9\%).

Figure. Antibody response to vaccination.

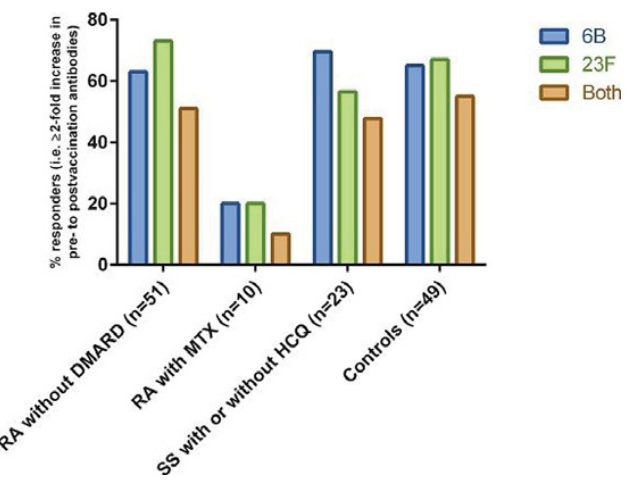

Conclusions: Pneumococcal conjugate vaccine is immunogenic in patients with $\mathrm{RA}$ and $\mathrm{pSS}$ without DMARD treatment. Both antibody response and functionality of antibodies is impaired in patients with RA on MTX.

Disclosure of Interest: None declared

DOI: 10.1136/annrheumdis-2017-eular.2146

Abstract FRI0162 - Table 1. Group characteristics and serotype-specific antibody geometric mean levels (GMLs, $\mu \mathrm{g} / \mathrm{mL}$ )

\begin{tabular}{|c|c|c|c|c|c|}
\hline & RA without DMARD $(n=51)$ & RA on $\operatorname{MTX}(n=10)$ & Sjogrens syndrome without DMARD $(n=18)$ & Sjogrens syndrome on HCQ $(n=5)$ & Controls $(n=49)$ \\
\hline Age (mean; range) years & $65.2(35-87)$ & $65.3(39-79)$ & $58.9(26-90)$ & $61.0(33-80)$ & $50.6(17-85)$ \\
\hline Sex (\% female) & 78.4 & 70.0 & 88.9 & 100.0 & 63.3 \\
\hline GML $6 \mathrm{~B}$ prevaccination $(95 \% \mathrm{Cl})$ & $0.6(0.4-1.0)$ & $1.3(0.5-2.9)$ & $0.6(0.2-1.5)$ & $0.6(0.2-1.7)$ & $0.8(0.5-1.3)$ \\
\hline GML $6 \mathrm{~B}$ postvaccination $(95 \% \mathrm{Cl})$ & $3.3(1.8-6.0)$ & $2.1(0.8-5.6)$ & $2.4(1.1-5.4)$ & $1.7(0.2-11.4)$ & $3.1(1.9-5.0)$ \\
\hline GML $23 \mathrm{~F}$ prevaccination $(95 \% \mathrm{Cl})$ & $0.5(0.3-0.8)$ & $1.0(0.3-3.5)$ & $0.8(0.4-1.6)$ & $0.4(0.2-1.2)$ & $0.6(0.4-0.9)$ \\
\hline GML $23 \mathrm{~F}$ postvaccination $(95 \% \mathrm{Cl})$ & $2.4(1.5-3.9)$ & $1.7(0.7-3.9)$ & $3.6(1.4-9.1)$ & $1.1(0.2-6.6)$ & $3.3(2.0-5.5)$ \\
\hline
\end{tabular}

